\title{
Platelet adhesiveness, plasma fibrinogen, and fibrinolytic activity in young patients with ischaemic stroke
}

\author{
S U B H A S H C. SH A R M A G. P. V I J A Y N, H. N. SETH A D \\ M. L. S U R I \\ From the Army Hospital, Delhi, India
}

SUMMARY Cerebral thromboembolic stroke in the young is uncommon. Disturbances in the normally existing dynamic equilibrium between coagulation and fibrinolysis may play a major role in the pathogenesis of such episodes. In a search for a possible hypercoagulable state platelet adhesiveness by the method of Eastham, plasma fibrinogen and euglobulin clot lysis times were estimated in 46 patients. A group of 15 normal subjects matched for age and sex were also studied for comparison. Significant increase in platelet adhesiveness $(P<0.001)$ and plasma fibrinogen $(\mathrm{P}<0.001)$ was noted in the patients when compared with the normal subjects. Absence of corresponding increases in fibrinolytic activity in the patients suggests disturbed equilibrium between coagulation and fibrinolysis. The possibility of a hypercoagulable state in these cases is discussed.

Despite increasing knowledge of thrombus formation and the blood fibrinolytic system, the importance of these in relation to the pathogenesis of thrombotic occlusion of cranial arteries is not fully understood. Although atherosclerosis of extracranial and intracranial arteries plays the principal aetiological role in thrombotic cerebrovascular diseases, it is likely, in view of Astrup's (1956) hypothesis, that the disequilibrium of the delicate balance existing between coagulation and fibrinolysis may determine the onset of thrombosis in many individuals. Platelets play a central role in coagulation and thrombogenesis. Elevated platelet adhesiveness (PA) in thromboembolic diseases has been reported by some investigators but not confirmed by others (Kirby and Martin, 1966; Bygdeman and Wells, 1969; Mason and Summerlin, 1972). A significant increase in PA in cerebrovascular thrombosis has been reported (Bedi et al., 1974; Sharma et al., 1977). It has also been suggested that the increase in plasma fibrinogen may predispose to thrombus formation by increasing the blood viscosity (Wells and Merrill, 1961; Burch and DePasquale, 1962). Hence it is logical to assume that the altered

Address for reprint requests: Major S. C. Sharma, Department of Pathology, AIIMS, New Delhi-110016, India.

Accepted 20 September 1977 fibrinolytic activity in such susceptible individuals is likely to be decisive in the final outcome.

Stroke in the young is considered uncommon (Berlin et al., 1955; Mayer, 1975). Results of recent studies suggest that there is an unusually high incidence of stroke at a younger age in the Indian population (Abraham et al., 1971; Sharma et al., 1977). The aetiology of ischaemic stroke in these individuals in whom the usual risk factors are lacking is still uncertain. Investigations on blood coagulation in thrombotic disorders have offered the possibility of detecting a hypercoagulable state (Murphy and Mustard, 1962; Ettinger, 1964). This claim, however, has not been substantiated by others (Mayer, 1975). The present study was undertaken in a search for the existence or otherwise of such a state in our patients, who represent a fair cross-section of the Indian population. Platelet adhesiveness plasma fibrinogen, and euglobulin clot lysis time (ELT) were estimated in 46 consecutive patients admitted to this centre. A group of 15 normal subjects matched for age and sex was also studied for comparison.

\section{Subjects and methods}

Sixty-one subjects comprising 46 patients with cerebral thromboembolism (group A) and 15 nor- 
mal individuals (group B) were studied. All were male in the age group 26 to 48 years.

The criteria for selection of cases were based on the report of the Advisory Council for the National Institute of Neurological Diseases and Blindness (Report by an ad hoc committee, 1964). Cases of haemorrhagic stroke were excluded by examination of the cerebrospinal fluid. None of the patients had clinical or laboratory evidence of hypertension, diabetes mellitus, syphilis, or any cardiac abnormality. Routine haemogram, urine analysis, ECG, EEG, and radiographs of skull, chest, and cervical spine were carried out in each patient. Angiographic studies of the appropriate vessels were performed to detect occlusive vascular diseases and to exclude conditions which may mimic cerebral ischaemic stroke, such as spaceoccupying lesions, aneurysms, and arteriovenous malformations.

Platelet adhesiveness was estimated by the method of Eastham (1964) using adenosine diphosphate (ADP). Two ml fasting blood was collected in siliconised syringes in the fasting state and was added to $2.4 \mathrm{mg}$ of ethylenediamine-tetra-acetic acid (EDTA), and $2 \mathrm{mg}$ of heparin in separate polystyrene bottles, and mixed in a Matburn mixer at $30 \mathrm{rev} / \mathrm{min}$. To the heparinised sample $0.04 \mathrm{ml}$ of ADP solution was added (from a stock solution of $5 \mathrm{mg}$ in $20 \mathrm{ml}$ saline), and bottles were returned to the mixer. After exactly $30 \mathrm{~min}$ the ADPtreated sample was transferred to another polystyrene bottle containing $2.4 \mathrm{mg}$ EDTA. Samples were mixed for $30 \mathrm{~min}$, and platelet counts were performed as soon as possible but in all cases within an hour of collection. Counts were performed in duplicate, and the results were averaged. The platelet adhesiveness was expressed as a percentage as follows:

Total EDT A sample count-

$$
\text { heparinised sample ADP count }
$$

$$
\text { Total EDTA sample count } \times 100
$$

Bearing in mind that factors such as diet (Philp and Wright, 1965, physical exertion (Pegrum et al., 1967), and smoking (Ashby et al., 1965) affect platelet adhesiveness, the procedure was standard- ised as rigidly as was practicable. All subjects were admitted to hospital. Normal subjects (group B) were detained in the hospital for at least four days for stabilisation before the tests, and rested in bed for most of this time. All the subjects had fasted overnight, and no smoking was permitted for three hours before venepuncture. No drug known to affect platelet behaviour was administered. Patients (group A) were studied usually three to four weeks after the onset of an acute episode, firstly because most of them were admitted as transfer cases from the peripheral hospitals, and secondly to eliminate any abnormalities resulting from thromboembolic phenomena. Plasma fibrinogen was estimated by the method of Ratnoff and Menzie (1951) and euglobulin clot lysis time by the method of Cash (1966). Statistical analysis was carried out using Student's $t$ test.

\section{Results}

Age, PA, plasma fibrinogen, ELT, and results of statistical analysis in respect of each group studied are given in the Table. Average age of the patients and control subjects was 32.5 and 33.4 years respectively. Most $(97.2 \%)$ of the patients were between 24 and 40 years old. The mean PA of controls and patients was $60.5 \%$ and $74.1 \%$ respectively, and the difference between them was statistically significant $(P<0.001)$. Plasma fibrinogen was also significantly $(\mathrm{P}<0.001)$ higher in the patients than in the normal subjects. The difference in ELT between the two groups was not significant $(P>0.05)$.

\section{Discussion}

Throughout his life man is almost constantly bleeding and thrombosing (Wright, 1952). Without thrombosis life would be impossible but thrombosis at the wrong place leads to death. Virchow (1863) was probably the first to introduce the concept of thromboembolism, and he laid stress on three factors: (a) slowing of the blood stream, (b) changes in the vessel wall, and (c) changes in the

Table Platelet adhesiveness $(P A)$, fibrinogen, and euglobulin lysis time $(E L T)$ in young patients with

\begin{tabular}{|c|c|c|c|c|}
\hline & $\begin{array}{l}\text { Age (range) } \\
(y r)\end{array}$ & $\begin{array}{l}P A \\
(\%)\end{array}$ & $\begin{array}{l}\text { Fibrinogen } \\
(m g / d l)\end{array}$ & $\begin{array}{l}E L T \\
(m i n)\end{array}$ \\
\hline $\begin{array}{l}\text { Control }(n=15) \\
\text { Patients }(n=46) \\
P \text { value control subjects versus patients }\end{array}$ & $\begin{array}{l}32.5(25-48) \\
33.4(24-45) \\
-\end{array}$ & $\begin{array}{l}60.5 \pm 5.8 \\
74.1 \pm 7.7 \\
<0.001\end{array}$ & $\begin{array}{l}252.1 \pm 33.1 \\
379.8 \pm 120.2 \\
<0.001\end{array}$ & $\begin{array}{l}302.8 \pm 57.6 \\
288.3 \pm 94.3 \\
\text { Not significant }\end{array}$ \\
\hline
\end{tabular}
ischaemic stroke.

Values are means $\pm S D ; n$ is the number of subjects. 
blood itself. Of these the last is perhaps the most important and the least understood. Normal fibrinolytic mechanisms are gradually being recognised to be of fundamental physiological importance. Formation of fibrin thrombi appears to be a regular phenomenon in the healthy human being. These deposits are constantly removed by the normal fibrinolytic system of the blood (Wright, 1952). Only limited information is currently available regarding abnormalities of platelet function and fibrinolytic system in thromboembolic stroke in the young.

PLATELET ADHESIVENESS (PA)

Our results indicate that patients with stroke have significantly higher $(\mathrm{P}<0.001) \mathrm{PA}$ than control subjects. Platelet adhesion appears to be one of the most important links in the sequence of changes in the circulating blood and the development of thrombosis, especially arterial thrombosis. In recent years interest has been focused on platelet adhesiveness in various diseases (Kirby and Martin, 1966; Bygdeman and Wells, 1969; Mason and Summerlin, 1972; Bedi et al., 1974). Despite discrepancies between the results presented by different authors it is very likely that the increased PA predisposes to thrombosis.

The time gap between the onset of stroke and the performance of tests does not affect the measured platelet adhesiveness (Danta, 1970; Acheson et al., 1972). Danta (1970) also came to the conclusion that PA in cases of stroke does not change significantly in the six weeks after the event. We, therefore, considered that PA as estimated three to four weeks after the stroke would reflect a stabilised state. Our limited experience, based on a small number of patients admitted directly to this centre after the acute episode and not on any chemotherapeutic agents likely to affect the platelet function, shows no significant change in the PA from the second to the ninth week (unpublished data), and is in partial agreement with the above reports. Controlled serial studies in a large number of patients are, however, required to delineate clearly the pattern of platelet function in the post-stroke period.

\section{PLASMA FIBRINOGEN}

Our data reveal a significant $(P<0.001)$ increase in plasma fibrinogen level in patients with stroke. Increase in plasma fibrinogen has been reported in stroke (Eisenburg, 1964; Ettinger et al., 1969; Gaston et al., 1971; Todd et al., 1973). It has been suggested that the elevated fibrinogen in the plasma will greatly increase the viscosity of blood and predispose to thrombus formation (Wells and Merrill, 1961; Burch and DePasquale, 1962). The increase in plasma fibrinogen in patients with coronary thrombosis is not transient, and is, therefore, to be distinguished from the short-lived increase found in stress or tissue injury like burns, trauma, and infection. The increase is probably due to increased biosynthesis and an enhanced rate of turnover (Pilgram, 1968). Whether the enhanced plasma fibrinogen in cases of stroke also results in the generation of potentially occlusive intravascular deposits of fibrin-platelet thrombi will depend upon the balance between the rate of formation of fibrin and the rate of its metabolism or lysis.

EUGLOBULIN LYSIS TIME (ELT)

Fibrinolytic activity in blood depends to a great extent upon the balance between plasminogen activators and inhibitors. Despite the criticism of substrate variability, the ELT is perhaps the best available method of assaying circulating plasminogen activator (Fletcher et al., 1964; Fearnley, 1965). Our data reveal no significant difference in ELT between patients with stroke and normal individuals. However, the role of the fibrinolytic system in the pathogenesis and the recovery stage of thromboembolic disorders is not yet clearly understood. Based on a study on 144 patients with nonhaemorrhagic stroke, Ettinger (1973) suggested that those having a rapid and strong fibrinolytic system stand a better chance of recovery.

Most $(97.2 \%)$ patients in the present study were young. The onset of ischaemic stroke in these younger individuals, in whom predisposing factors such as diabetes mellitus, hypertension, ischaemic, and valvular heart diseases have been excluded, suggests that significant atherosclerotic lesions in the cranial vessels are unlikely to be a major contributory factor. Our own observations on angiographic studies in these patients also favour this conclusion. Thus a dynamic equilibrium between coagulation and fibrinolysis and a significant increase in platelet adhesiveness and plasma fibrinogen level, in the face of no corresponding increase in the fibrinolytic activity, probably point towards a state of "hypercoagulability". It is likely that the disequilibrium predisposed these patients to thrombosis. Long-term studies on a larger number of subjects are, however, required for the better understanding of this situation.

\section{References}

Abraham, J., Shetty, G., and Jose, C. J. (1971). Stroke in the young. Stroke, 2, 258-267.

Acheson, J., Danta, G., and Hutchinson, E. C. (1972). 
Platelet adhesiveness in patients with cerebral vascular disease. A therosclerosis, 15, 123-127.

Ashby, P., Dalby, A. M., and Millar, J. H. D. (1965). Smoking and platelet stickiness. Lancet, 2, 158-159.

Astrup, T. (1956). Biological significance of fibrinolysis. Lancet, 2, 565-568.

Bedi, H. K., Bomb, B. S.. Devpura, J. C. Vyas, B. R., and Bedi, T. (1974). Platelet adhesiveness in cerebral thrombosis. Journal of the Association of Physicians of India, 22, 829-831.

Berlin, L., Tumarkin, B., and Martin, H. L. (1955). Cerebral thrombosis in young adults. New England Journal of Medicine, 252, 162-166.

Burch. G. E., and DePasquale, N. P. (1962). Hematocrit, blood viscosity and myocardial infarction. (Editorial) American Journal of Medicine, 32, 161163.

Bygdeman, S., and Wells, R. (1969). Studies of platelet viscosity and the microcirculation in patients with thrombotic diseases. Journal of Atherosclerosis Research, 10, 33-39.

Cash, J. D. (1966). Effect of moderate exercise on the fibrinolytic system in normal young men and women. British Medical Journal, 2, 502-505.

Danta, G. (1970). Platelet adhesiveness in cerebrovascular disease. Atherosclerosis, 11, 223-233.

Eastham, R. D. (1964). Rapid adhesive platelet count in whole blood. Journal of Clinical Pathology, 17, 45-46.

Eisenburg. S. (1964). Blood viscosity and fibrinogen concentration following cerebral infarction. Circulation, 33 (Suppl. 11), 10-14.

Ettinger, M. G. (1964). Coagulation studies in cerebrovascular disease, Part I (hypercoagulability). Neurology (Minneapolis), 14, 907-911.

Ettinger, M. G. (1973). Thrombolytic studies in cerebrovascular disease. Prediction of recovery from neurological deficit following cerebral infarction. Neurology (Minneapolis), 23, 397.

Ettinger, M. G., Kusonoki, R., Fusishima, H. (1969). Blood coagulation studies. Geriatrics, 24, 116-125.

Fearnley, G. R. (1965). Fibrinolysis, p. 35. Edward Arnold: London.

Fletcher, A. P., Biederman, O., Moore, D., Alkjaarsig. N.. and Sherry, S. (1964). Abnormal plasminogen-plasmin system activity (fibrinolysis) in patients with hepatic cirrhosis: its cause and consequences. Journal of Clinical Investigations, 43, 681-695.

Gaston. L. W., Brooks. J. E., Blumenthal. H. J.. and
Muller, C. E. (1971). A study of blood coagulation following acute stroke. Stroke, 2, 81-87.

Kirby, J. C., and Martin, C. L. (1966). Platelet adhesiveness and vascular disease. Circulation, 34 (Suppl. III), 17.

Mason, R. G., and Summerlin, D. C. (1972). Alterations of platelet adhesion to glass in vascular disorders and certain other diseases. American Journal of Clinical Pathology, 57, 611-617.

Mayer, J. S. (1975). Summary of the international round table conference on platelet aggregation in the pathogenesis of cerebrovascular disorders. Stroke, 6, 239-244.

Murphy, E. A.. and Mustard, J. F. (1962). Coagulation tests and platelet economy in atherosclerotic and control subjects. Circulation, 25, 114-125.

Pegrum, G. D., Harrison, K. M., Shaw, S., Haselton, A., and Wolff, S. (1967). Effect of prolonged exercise on platelet adhesiveness. Nature, 213, 301-302.

Philp, R. B., and Wright, H. P. (1965). Effects of adenosine on platelet adhesiveness in fasting and lipaemic bloods. Lancet, 2, 208-209.

Pilgram, L. O. (1968). Turnover rate of autologous plasma fibrinogen $\mathrm{C}-14$ in subjects with coronary thrombosis. Thrombosis et Diathesis Haemorrhagica, 20, 31-43.

Ratnoff, O. D., and Menzie, C. (1951). A new method of determination of fibrinogen in small samples of plasma. Journal of Laboratory and Clinical Medicine, 37, 316-320.

Report by an ad hoc committee. (1964). A classification and outline of cerebrovascular disease. Neurology (Minneapolis), 8, 397-434.

Sharma, S. C., Vijayan, G. P., Suri, M. L., and Seth. H. N. (1977). Platelet adhesiveness in young patients with ischaemic stroke. Journal of Clinical Pathology, 36, 649-652

Todd, M.. McDevitt, E., and McDowell, F. (1973). Stroke and blood coagulation. Stroke, 4, 400-405.

Virchow, R. (1863). Cellular Pathology. As based upon physiological and pathological histology. Translated from the second German edition originally published by J. B. Lippincott and Co New York. Dover Pubns. Inc: New York. 1971.

Wells, R. E., and Merrill, E. W. (1961). The variability of blood viscosity. (Editorial) American Journal of Medicine. 31, 505-509.

Wright, I. S. (1952). Thrombosis. Circulation, 5, 161188. 